\title{
A Sufficient Condition for Matrix Stability*
}

\author{
Charles R. Johnson** \\ Institute for Basic Standards, National Bureau of Standards, Washington, D.C. 20234
}

(April 22, 1974)

\begin{abstract}
An $n$ by $n$ complex matrix $A$ is said to be positive stable if $\operatorname{Re}(\lambda)>0$ for each eigenvalue $\lambda$ of $A$. If $A$ satisfies both of the following two conditions, then $A$ is positive stable: (1) for each $k=1, \ldots ., n$, the real part of the sum of the $k$ by $k$ principal minors of $A$ is positive; and (2) the minimum of the real parts of the eigenvalues of $A$ is itself an eigenvalue of $A$. Special cases include hermitian positive definite matrices and $M$-matrices.
\end{abstract}

Key words: $M$-matrix; stable matrix; principal minors.

Suppose $A=\left(a_{i j}\right) \epsilon M_{n}(C)$, the $n$ by $n$ complex matrices. Then let $P_{k}(A)$ denote the sum of the $k$ by $k$ principal minors of $A$. If $\operatorname{Re}(\lambda)>0$ for each eigenvalue $\lambda$ of $A$, then $A$ is called (positive) stable, and if $\operatorname{Re}\left[P_{k}(A)\right]>0, k=1, \ldots, n$, then $A$ is called prestable [2]. ${ }^{1}$ This note was suggested by the following question which arose in the stability analysis of an economic equilibrium [3]. Suppose $A \epsilon M_{n}(R)$, the real $n$ by $n$ matrices, has the sign pattern $(M)$ :

and

$$
a_{i i}>0, \quad i=1, \ldots, n
$$

$$
a_{i j} \leqslant 0, \quad i \neq j, i, j=1, \ldots, n .
$$

If $\mathrm{A}$ is prestable, does it then follow that $\mathrm{A}$ is stable? Unfortunately, this is not one of the 13 equivalent conditions given in [1] for $A$ to be an $M$-matrix. An affirmative answer to this question is a corollary to what we shall prove.

LEMma 1: If $\mathrm{A}_{\mathrm{G}} \mathrm{M}_{\mathrm{n}}(\mathrm{C})$ is prestable, then $\mathrm{A}$ has no nonpositive real eigenvalues.

Proof: Suppose $A$ is prestable and that $-r, r \geqslant 0$, is a nonpositive real eigenvalue of $A$. But then $A-(-r) I=A+r I$ is singular which means $\operatorname{det}(A+r I)=0$. However,

$$
\operatorname{det}(A+r I)=r^{n}+\sum_{k=1}^{n} P_{k}(A) r^{(n-k)}
$$

so that $\operatorname{Re}[\operatorname{det}(A+r I)]>0$, a contradiction. This means there is no such $r$ and completes the proof.

When the minimum of the real parts of the eigenvalues of $A \epsilon M_{n}(C)$ is itself an eigenvalue of $A$, we shall say that $A$ has the property (*).

Theorem: Suppose $\mathrm{A}_{\mathrm{G}} \mathrm{M}_{\mathrm{n}}(\mathrm{C})$ has property $\left({ }^{*}\right)$. If $\mathrm{A}$ is prestable, then $\mathrm{A}$ is stable.

Proof: Because of lemma 1 and property $(*)$, the minimum of the real parts of the eigenvalues of $A$ is positive. This means $A$ is stable.

Since it is clear that the well known class of hermitian matrices satisfies property $\left(^{*}\right)$, it follows that:

AMS Subject Classification: 15-A18, 15-A42, 93-D05.

*This work was done while the author was a National Academy of Sciences-National Research Council Postdoctoral Research Associate at the National Bureau of Standards, Washington, D.C. 20234.

***Present address: Institute for Fluid Dynamics and Applied Mathematics, University of Maryland, College Park, Md. 20742.

${ }^{1}$ Figures in brackeis indicate the literature references at the end of this paper. 
Corollary 1: If $\mathrm{A} \in \mathrm{M}_{\mathrm{n}}(\mathrm{C})$ is hermitian, then $\mathrm{A}$ prestable implies $\mathrm{A}$ stable.

Another well known class of matrices which has property $\left(^{*}\right)$ are those of sign pattern $(M)$.

Lemma 2: If $\mathrm{A} \epsilon \mathrm{M}_{\mathrm{n}}(\mathrm{R})$ has sign pattern $(\mathrm{M})$, then A has property $\left({ }^{*}\right)$.

Proof: Suppose that $m$ is a real number greater than each of the $a_{i i}$. Then $A$ may be written

$$
A=m I-P
$$

where $P$ is an entry-wise nonnegative matrix. Because of the Perron-Frobenius theorem, $P$ has a dominant positive eigenvalue $r$ such that $r \geqslant|\lambda|$, and thus $r \geqslant \operatorname{Re}(\lambda)$, for each eigenvalue $\lambda$ of $P$. It then follows that $m-r$ is an eigenvalue of $A$ and is the smallest of the real parts of the eigenvalues of $A$, so that $A$ has property (*).

Corollary 2: If $\mathrm{A} \in \mathrm{M}_{\mathrm{n}}(\mathrm{R})$ has sign pattern (M), then A prestable implies A stable.

Proof: This follows from Lemma 2 and the theorem.

We close with a question of interest which arises from the preceding remarks:

What are necessary and sufficient conditions on A such that A satisfy property (*)?

\section{References}

[1] Fiedler, M., and Ptak, V., On matrices with nonpositive off-diagonal elements and positive principal minors, Czech. M. J. 12, 382-400 (1962).

[2] Maybee, J., in preparation.

[3] Quirk, J., Private communication, December 1973.

(Paper 78B3-405) 